\title{
Maximizing Wireless Network Capacity with Linear Power: Breaking The Logarithmic Barrier
}

\author{
Peng-Jun Wan*, Lei Wang ${ }^{\dagger}$, Chao Ma*, Zhu Wang*, Boliu $\mathrm{Xu}^{*}$, and Minming Li ${ }^{\ddagger}$ \\ * Department of Computer Science, Illinois Institute of Technology, wan@cs.iit.edu, \{cma3, zwang59, bxu8\}@iit.edu \\ $\dagger$ School of Software, Dalian University of Technology, lei.wang@ieee.org \\ $\ddagger$ Department of Computer Science, City University of Hong Kong, minming.li@cityu.edu.hk
}

\begin{abstract}
Maximizing the wireless network capacity under physical interference model is notoriously hard due to the nonlocality and the additive nature of the wireless interference under the physical interference model. This problem has been extensively studied recently with the achievable approximation bounds progressively improved from the linear factor to logarithmic factor. It has been a major open problem whether there exists a constant-approximation approximation algorithm for maximizing the wireless network capacity under the physical interference model. In this paper, we improve the status quo for the case of linear transmission power assignment, which is widely adopted due to its advantage of energy conservation. By exploring and exploiting the rich nature of the wireless interference with the linear power assignment, we develop constantapproximation algorithms for maximizing the wireless network capacity with linear transmission power assignment under the physical interference model, in both the unidirectional mode and the bidirectional mode.
\end{abstract}

Index Terms-Link scheduling, physical interference, approximation algorithms.

\section{INTRODUCTION}

Consider a multihop wireless network consisting of a planar set $V$ of networking nodes. Under the physical interference model, the signal strength attenuates with a path loss factor $\eta r^{-\kappa}$, where $r$ is the distance from the transmitter, $\kappa$ is pathloss exponent (a constant between 2 and 5 depending on the wireless environment), and $\eta$ is the reference loss factor. The signal quality perceived by a receiver is measured by the signal to interference and noise ratio (SINR), which is the quotient between the power of the wanted signal and the total power of unwanted signals (i.e., interferences) and the ambient noise $\xi$. In order to correctly interpret the wanted signal, the SINR must exceed certain threshold $\sigma$. Thus, for a communication from a node $u$ to a node $v$ to be possible even without any interference, the transmission power of $u$ should exceed $\frac{\sigma \xi}{\eta}\|u v\|^{\kappa}$, where $\|u v\|$ denotes the Eulcidean distance between $u$ and $v$. In general, for any ordered pair $a$ of nodes $(u, v)$ we use $\ell(a)$ to denote $\|u v\|$ and $p_{0}(a)$ to denote $\frac{\sigma \xi}{\eta} \ell(a)^{\kappa}$. Suppose that all nodes have maximum transmission power $P$. Then, the largest possible set of communication links, denoted by $A$, consists of all possible pairs $a$ of nodes satisfying that $p_{0}(a)<P$. For a specific power assignment $p$, the set of communication links, denoted by $A_{p}$, consists of all possible pairs $a$ of nodes satisfying that $p_{0}(a)<p(a) \leq P$. Clearly, $A_{p} \subseteq A$.
In this paper, we consider the linear power assignment $p$ given by $p(a)=c p_{0}(a)$ for some constant $c>1$. The linear power assignment is widely adopted due to its advantage of energy conservation. We also consider the following two communication modes of the physical interference model which have been considered in the literature:

- Unidirectional mode: In this mode, the communications between a pair of nodes are unidirectional, and the interference distance from a link $a$ to another link $b$, denoted by $\ell(a, b)$, is the distance between the sender of $a$ and the receiver of $b$.

- Bidirectional mode: In this mode, the communications between a pair of nodes are bidirectional, and the interference distance from a link $a$ to another link $b$, denoted by $\ell(a, b)$, is the shortest distance between the two endpoints of $a$ and the two endpoints of $b$.

For any pair of distinct links $a$ and $b$ in $A_{p}$, when they transmit at the same time the interference of $a$ toward $b$ is $p(a) \cdot \eta \ell(a, b)^{-\kappa}$. Let $\mathcal{I}_{p}$ denote the collection of all subsets of $A_{p}$ which can communicate successfully at the same time. Each set in $\mathcal{I}_{p}$ is referred to as an independent set. The maximum size of the independent sets is called the independence number of the network, and is denoted by $\alpha$.

Suppose that $d \in \mathbb{R}_{+}^{|A|}$ is a vector indexed by the links in A. A (fractional) link schedule of $d$ is a set

$$
\Pi=\left\{\left(I_{j}, l_{j}\right) \in \mathcal{I} \times \mathbb{R}_{+}: 1 \leq j \leq k\right\}
$$

satisfying that for each link $a \in A$,

$$
d_{a}=\sum_{j=1}^{k} \ell_{j}\left|I_{j} \cap\{a\}\right| ;
$$

the value $\sum_{j=1}^{k} l_{j}$ is referred to as length (or latency) of $\Pi$. If the length of $\Pi$ is one, then $d$ is also referred to as the link capacity vector determined by $\Pi$. The two fundamental cross-layer capacity optimization problems are Maximum Multiflow (MMF) and Maximum Concurrent Multiflow (MCMF) described as follows:

- Maximum Multiflow (MMF): Given a set of end-to-end communication requests specified by source-destination pairs, find a fractional link schedule $\Pi$ of length one such that the maximum multiflow subject to the link capacity vector determined by $\Pi$ is maximized. 
- Maximum Concurrent Multiflow (MCMF): Given a set of end-to-end communication requests specified by source-destination pairs together with their demands, find a fractional link schedule $\Pi$ of length one such that the maximum concurrent multiflow subject to the link capacity vector determined by $\Pi$ is maximized.

For both MMF and MCMF, the best-known approximation bounds are $O(\log \alpha)$ [13] (the explicit bounds are given in Section II). It has been a major open problem on the existence of polynomial constant-approximation algorithms for MMF and MCMF.

In this paper, we present polynomial constant-approximation algorithms for MMF and MCMF with linear power assignment. In the unidirectional mode, their approximation ratios are at most

$$
8\left(\left\lceil\pi / \arcsin \frac{1-\left(\frac{c-1}{c \sigma}\right)^{1 / \kappa}}{2}\right\rceil-1\right) ;
$$

In the bidirectional mode, their approximation ratios are at most 80 . These constant-approximation algorithms exploit the rich nature of wireless interference with the linear power assignment discovered in this paper. Such rich nature is also exploited to develop improved approximation algorithms for other related problems described in Section II.

The remaining of this paper is organized as follows. In Section II, we introduce some related problems and prior works. In Section III, we explore the rich nature of wireless interference with the linear power assignment. In Section IV, we present the design and analysis of our constant-approximation algorithms. Finally we conclude in this paper in Section $\mathrm{V}$ by discussion on the challenges to achieving constantapproximation bounds for other monotone and sublinear power assignments.

\section{Related Problems ANd Works}

Both MMF and MCMF are closely related to the following simpler MAC-layer only wireless link scheduling problems under an arbitrary fixed power assignment $p$ :

- Maximum Independent Set of Links (MISL): Given a subset $B$ of $A_{p}$, find a largest indepdent subset of $B$.

- Maximum Weighted Independent Set of Links (MWISL): Given a link weight vector $w \in \mathbb{R}_{+}^{\left|A_{p}\right|}$, find an $I \in \mathcal{I}_{p}$ with maximum total weight $\sum_{a \in I} w_{a}$.

- Shortest Fractional Link Schedule (SFLS): Given a link demand vector $d \in \mathbb{R}_{+}^{\left|A_{p}\right|}$, find a shortest fractional link schedule of $d$.

The following two theorems characaterizing their relations were established in [13], [16].

Theorem 2.1: If there is a polynomial $\mu$-approximation) algorithm for MWISL, then all of MMF, MCMF and SFLS have a polynomial $\mu$-approximation algorithm.

Theorem 2.2: If MISL has a polynomial $\mu$-approximation algorithm, then SFLS has a polynomial $(1+\mu \ln \alpha)$ approximation algorithm, and all of the three problems MWISL, MMF, and MCMF all have a polynomial $e \mu(1+\ln \alpha)$-approximation algorithm, where $e \approx 2.718$ is the natural base.

Constant-approximation algoprithms for MISL with various power assignments have been developed in [7], [9], [12], [14]. Among them, the best-known approximation bounds for MISL with linear power asignment are $960 \cdot 3^{\kappa}$ in the unidirectional mode [7], and $80\left(\frac{3}{2}+\sqrt{2}\right)$ in the bidirectional mode [12]. Consequently, we have the following best-known approximation bounds for other problems with linear power assignment:

- In the unidirectional mode, the best-known approximation bound for SFLS is

$$
1+960 \cdot 3^{\kappa} \ln \alpha,
$$

and the best-known approximation bounds for MWISL, MMF, and MCMF are

$$
960 \cdot 3^{\kappa} e(1+\ln \alpha) .
$$

- In the bidirectional mode, the best-known approximation bounds for SFLS is

$$
1+80\left(\frac{3}{2}+\sqrt{2}\right) \ln \alpha
$$

and the best-known approximation bounds for MWISL, MMF, and MCMF are

$$
80\left(\frac{3}{2}+\sqrt{2}\right) e(1+\ln \alpha) .
$$

Recently, Halldórsson and Mitra [8] developed a randomized constant-approximation algorithm for MWISL with linear power assignment in the unidirectional mode. However, the approximation-preserving reductions from MMF, MCMF and SFLS to MWISL established in Theorem 2.1 are only valid for deterministic algorithms. Thus, the randomized algorithm for MWISL in [8] cannot imply even randomized constantapproximation algorithms for MMF, MCMF and SFLS, let alone deterministic constant-approximation algorithms for MMF, MCMF and SFLS.

The vairants of wireless link scheduling with power control have been studied in [1], [4], [6], [7], [10], [12], [15], [16].

\section{INWARD LOCAL INDEPENDENCE NUMBER}

Consider a linear power assignment $p$ to $A$ given by $p(a)=$ $c p_{0}(a)$ for some constant $c>1$. For any two distinct links $a, b \in A_{p}$, the relative interference of $a \in A_{p}$ toward $b$ is defined to be

$$
R I_{p}(a, b)=\left(\frac{\ell(a)}{\varepsilon \ell(a, b)}\right)^{\kappa}
$$

where

$$
\varepsilon=\left(\frac{c-1}{c \sigma}\right)^{1 / \kappa}
$$

and the capped relative interference from $a$ toward $b$ is defined to be

$$
\overline{R I}_{p}(a, b)=\min \left\{1, R I_{p}(a, b)\right\} .
$$


In general, for any pair of disjoint subsets $B_{1}$ and $B_{2}$ of $A_{p}$, the relative interference from $B_{1}$ toward $B_{2}$ is defined to be

$$
R I_{p}\left(B_{1}, B_{2}\right)=\sum_{b_{1} \in B_{1}} \sum_{b_{2} \in B_{2}} R I_{p}\left(b_{1}, b_{2}\right) .
$$

and the capped relative interference from $B_{1}$ toward $B_{2}$ is defined to be

$$
\overline{R I}_{p}\left(B_{1}, B_{2}\right)=\sum_{b_{1} \in B_{1}} \sum_{b_{2} \in B_{2}} \overline{R I}_{p}\left(b_{1}, b_{2}\right) .
$$

It is easy to verify that for any subset $I$ of $A_{p}$,

$$
\begin{aligned}
I \in \mathcal{I}_{p} & \Leftrightarrow \max _{a \in I} R I_{p}(I \backslash\{a\}, a)<1 \\
& \Leftrightarrow \max _{a \in I} \overline{R I}_{p}(I \backslash\{a\}, a)<1 .
\end{aligned}
$$

The inward local independence number (ILIN) of the power assignment $p$ is defined to be

$$
\beta_{p}=\max \left\{\overline{R I}_{p}(I \backslash\{a\}, a): I \in \mathcal{I}_{p}, a \in A_{p}\right\} .
$$

In this section, we derive the following upper bounds on $\beta_{p}$.

Theorem 3.1: $\beta_{p}$ is less than 20 in the bidirectional mode, and less than $2\left(\left\lceil\pi / \arcsin \frac{1-\varepsilon}{2}\right\rceil-1\right)$ in the unidirectional mode.

To prove the above theorem, we introduce the notion of guarding number of the power assignment $p$. Consider a set $B$ of disjoint links in $A$ and a link $a \in A$ and. A subset $S$ of $B$ is said to be a guarding subset of $B$ from $a$ if for each link $b \in B \backslash S$,

$$
\min _{a^{\prime} \in S} \ell\left(b, a^{\prime}\right) \leq \ell(b, a) .
$$

A minimum guarding subset of $B$ from $a$ is a guarding subset of $B$ from $a$ which has the smallest size, and its size is denoted by $\gamma(B, a)$. The guarding number of the power assignment $p$ is defined to be to be

$$
\gamma_{p}=\max \left\{\gamma(I, a): I \in \mathcal{I}_{p}, a \in A_{p} \backslash I\right\} .
$$

Then, the two parameters $\beta_{p}$ and $\gamma_{p}$ have the following relation.

Lemma 3.2: $\beta_{p}<2 \gamma_{p}$.

Proof: Let $I \in \mathcal{I}_{p}$ and $a \in A_{p}$ be such that $\beta_{p}=$ $\overline{R I}_{p}(I \backslash\{a\}, a)$. If $a \in I$, then $\beta_{p}<1<2 \gamma_{p}$ since $\gamma_{p} \geq 1$. So we assume that $a \notin I$.

Let $S$ be a minimum guarding subset of $I$ from $a$. We claim that for any link $b \in I \backslash S, R I_{p}(b, a) \leq R I_{p}(b, S)$. Indeed, let $a^{\prime} \in S$ be such that $\ell(b, a) \geq \ell\left(b, a^{\prime}\right)$. Since $a^{\prime}$ and $b$ are disjoint, $\ell\left(b, a^{\prime}\right)>0$ and hence $\ell(b, a)>0$. So,

$$
\frac{R I_{p}(b, a)}{R I_{p}\left(b, a^{\prime}\right)}=\left(\frac{\ell\left(b, a^{\prime}\right)}{\ell(b, a)}\right)^{\kappa} \leq 1
$$

Thus,

$$
R I_{p}(b, a) \leq R I_{p}\left(b, a^{\prime}\right) \leq R I_{p}(b, S) .
$$

So, the claim holds.

The above claim implies

$$
\overline{R I}_{p}(I \backslash S, a) \leq R I_{p}(I \backslash S, a) \leq R I_{p}(I \backslash S, S)<|S| .
$$

Thus,

$$
\begin{aligned}
\beta_{p} & =\overline{R I}_{p}(S, a)+\overline{R I}_{p}(I \backslash S, a)<|S|+|S| \\
& =2|S|=2 \gamma(I, a) \leq 2 \gamma_{p} .
\end{aligned}
$$

So, the lemma holds.

By Lemma 3.2, in order to prove Theorem 3.1 it is sufficient to establish the following bounds on $\gamma_{p}$.

Theorem 3.3: $\gamma_{p}$ is at most 10 in the bidirectional mode, and at most $\left[\pi / \arcsin \frac{1-\varepsilon}{2}\right]-1$ in the unidirectional mode.

The upper bound 10 on $\gamma_{p}$ in the bidirectional mode was already proven in Lemma 4.4 of [12], which states a more general result that $\gamma(B, a) \leq 10$ for any $B \subseteq A$ and any $a \in A$. In the remaining of this section, we assume the unidirectional mode and prove that

$$
\gamma_{p} \leq\left\lceil\pi / \arcsin \frac{1-\varepsilon}{2}\right\rceil-1
$$

using an angular argument based on the following geometric lemma, whose proof is omitted due to the space limit.

Lemma 3.4: Consider a triangle $\triangle o w v$ with $\|o v\| \geq\|o w\|$ and

$$
\angle \text { vow } \leq 2 \arcsin \frac{1-\varepsilon}{2},
$$

for some $0<\varepsilon<1$. Then for any point $u$,

$$
\|u w\| \leq \max \{\|o u\|,\|u v\| / \varepsilon\} .
$$

Let $I \in \mathcal{I}_{p}$ and $a \in A_{p} \backslash I$ be such that $\gamma_{p}=\gamma(I, a)$. Let $o$ be the receiver of $a$. If $o$ is also the receiver of some link $a^{\prime}$ in $I$, then for any link $b \in I, \ell(b, a)=\ell\left(b, a^{\prime}\right)$; hence $\left\{a^{\prime}\right\}$ is a guarding subset of $I$ from $a$ and consequently

$$
\gamma_{p}=\gamma(I, a) \leq 1 \leq\left\lceil\pi / \arcsin \frac{1-\varepsilon}{2}\right\rceil-1 .
$$

So, we assume that $o$ is not the receiver of any link in $I$. We present a greedy construction of a guarding subset $S$ of $I$ from $a$. Initially, $S$ is set to empty and $I^{\prime}$ is set to $I$. Repeat the following iterations while $I^{\prime}$ is non-empty: Let $a^{\prime}$ be a link in $I^{\prime}$ whose receiver is closest to $o$, add $a^{\prime}$ to $S$, and remove $a^{\prime}$ and all other links $b$ in $I^{\prime}$ satisfying that $\ell(b, a) \geq \ell\left(b, a^{\prime}\right)$. Clearly, the output set is a guarding subset of $I$ to $a$. We shall show that

$$
|S| \leq\left\lceil\pi / \arcsin \frac{1-\varepsilon}{2}\right\rceil-1,
$$

from which we have

$$
\gamma_{p}=\gamma(I, a) \leq|S| \leq\left\lceil\pi / \arcsin \frac{1-\varepsilon}{2}\right\rceil-1 .
$$

We first claim that for any pair of links $a_{1}=\left(u_{1}, v_{1}\right)$ and $a_{2}=\left(u_{2}, v_{2}\right)$ in $S, \angle v_{1} o v_{2} \leq 2 \arcsin \frac{1-\varepsilon}{2}$. Assume to the contrary that the claim does not hold. By symmetry, we assume that $a_{1}$ is added to $S$ before $a_{2}$. Then, $\left\|o v_{1}\right\| \leq\left\|o v_{2}\right\|$. By Lemma 3.4,

$$
\begin{aligned}
\ell\left(a_{2}, a_{1}\right) & =\left\|u_{2} v_{1}\right\| \leq \max \left\{\left\|o u_{2}\right\|,\left\|u_{2} v_{2}\right\| / \varepsilon\right\} \\
& =\max \left\{\ell\left(a_{2}, a\right), \ell\left(a_{2}\right) / \varepsilon\right\} .
\end{aligned}
$$


Since

$$
1>R I_{p}\left(a_{2}, a_{1}\right)=\left(\frac{\ell\left(a_{2}\right)}{\varepsilon \ell\left(a_{2}, a_{1}\right)}\right)^{\kappa}
$$

we have $\ell\left(a_{2}, a_{1}\right)>\ell\left(a_{2}\right) / \varepsilon$, and hence $\ell\left(a_{2}, a\right) \geq$ $\ell\left(a_{2}, a_{1}\right)$. Thus, $a_{2}$ should have been removed from from $I^{\prime}$ after the iteration in which $a_{1}$ is added to $S$, which is a contradiction. So, our claim holds.

The claim in the previous paragraph immediately implies that

$$
|S| \leq\left\lceil\pi / \arcsin \frac{1-\varepsilon}{2}\right\rceil-1
$$

This completes the proof of Theorem 3.3.

\section{Scheduling Algorithms}

In this section, we shall establish the following main theorem of this paper.

Theorem 4.1: All of MWISL, MMF, MCMF and SFLS have a polynomial $4 \beta_{p}$-approximation algorithm.

By Theorem 3.1, the approximation bound $4 \beta_{p}$ is less than 80 in the bidirectional mode, and less than $6\left(\left\lceil\pi / \arcsin \frac{1-\varepsilon}{2}\right\rceil-1\right)$ in the unidirectional mode. Since MISL is a special case of MWISL, a polynomial $4 \beta_{p^{-}}$ approximation algorithm for MWISL also yields a polynomial $4 \beta_{p}$-approximation algorithm for MISL. For MISL, The approximation bound $4 \beta_{p}$ beats the previously best-known approximation bound $80\left(\frac{3}{2}+\sqrt{2}\right)$ in the bidirectional mode [12], and $960 \cdot 3^{\kappa}$ in the unidirectional mode [7].

Now, we present an algorithm LPWIS for MWISL. Suppose that $w \in \mathbb{R}_{+}^{\left|A_{p}\right|}$ is a link-weight vector indexed by the links in $A_{p}$. The algorithm runs in three phases as described below.

Phase 1: Compute an optimal solution $x$ to the following linear program:

$$
\begin{array}{ll}
\max & \sum_{a \in A_{p}} w_{a} x_{a} \\
\text { s.t. } & \sum_{a \in A_{p} \backslash\{b\}} \overline{R I}_{p}(a, b) x_{a} \leq \frac{1}{2}, \forall b \in A_{p} \\
& 0 \leq x_{a} \leq 1, \forall a \in A_{p} .
\end{array}
$$

Phase 2: Round $x$ to integers. Set

$$
B=\left\{a \in A_{p}: 0<x_{a}<1\right\} .
$$

Repeat the following iterations until $B$ is non-empty: Pick a link $a \in B$ and remove it from $B$. If

$w_{a}\left(1-\sum_{b \in A_{p} \backslash\{a\}} \overline{R I}_{p}(b, a) x_{b}\right)>\sum_{b \in A_{p} \backslash\{a\}} \overline{R I}_{p}(a, b) w_{b} x_{b}$, set $x_{a}=1$; otherwise set $x_{a}=0$.

Phase 3: Compute an independent set $I$. Initialize $I$ to an empty set. For each $a \in A_{p}$, if $x_{a}=1$ and

$$
\sum_{b \in A_{p} \backslash\{a\}} \overline{R I}_{p}(b, a)<1
$$

then add $a$ to $I$. Finally, output $I$.

The theorem below proves the correctness of the LPWIS and analyzes its performance.
Theorem 4.2: Let $I$ be the output by the algorithm LPWIS, and opt the weight of a maximum weighted independent set in $\mathcal{I}_{p}$. Then, $I \in \mathcal{I}_{p}$ and

$$
\sum_{a \in I} w_{a} \geq \frac{1}{4 \beta_{p}} \text { opt }
$$

Proof: We define a function $f$ from the set of vectors $x \in[0,1]^{\left|A_{p}\right|}$ indexed by the set of links in $A_{p}$ to real numbers by

$$
f(x)=\sum_{a \in A_{p}} w_{a} x_{a}\left(1-\sum_{b \in A_{p} \backslash\{a\}} \overline{R I}_{p}(b, a) x_{b}\right) .
$$

Then for each $a \in A_{p}, f$ is a linear function of $x_{a}$ and $\frac{\partial f(x)}{\partial x_{a}}$ is

$w_{a}\left(1-\sum_{b \in A_{p} \backslash\{a\}} \overline{R I}_{p}(b, a) x_{b}\right)-\sum_{b \in A_{p} \backslash\{a\}} \overline{R I}_{p}(a, b) w_{b} x_{b}$.

We first show that at the end of Phase 1,

$$
f(x) \geq \frac{1}{4 \beta_{p}} \text { opt }
$$

Let $O$ be a maximum weighted independent set. Define a vector $y \in[0,1]^{\left|A_{p}\right|}$ by

$$
y_{a}= \begin{cases}\frac{1}{2 \beta_{p}}, & \text { if } a \in O \\ 0, & \text { otherwise }\end{cases}
$$

Then, $y$ is a feasible solution to the linear program defined in Phase 1 as for each $b \in A_{p}$,

$$
\begin{aligned}
& \sum_{a \in A_{p} \backslash\{b\}} \overline{R I}_{p}(a, b) y_{a}=\frac{1}{2 \beta_{p}} \sum_{a \in O \backslash\{b\}} \overline{R I}_{p}(a, b) \\
= & \frac{1}{2 \beta_{p}} \overline{R I}_{p}(O \backslash\{b\}, b) \leq \frac{1}{2 \beta_{p}} \beta_{p}=\frac{1}{2}
\end{aligned}
$$

and its value is

$$
\sum_{a \in A_{p}} w_{a} y_{a}=\frac{1}{2 \beta_{p}} \sum_{a \in O} w_{a}=\frac{1}{2 \beta_{p}} \text { opt. }
$$

Since $x$ is an optimal solution to the same linear program,

$$
\sum_{a \in A_{p}} w_{a} x_{a} \geq \frac{1}{2 \beta_{p}} \text { opt. }
$$

Since for each $a \in A_{p}$,

$$
1-\sum_{b \in A_{p} \backslash\{a\}} \overline{R I}_{p}(b, a) x_{b} \geq 1-\frac{1}{2} \geq \frac{1}{2},
$$

we have

$$
\begin{aligned}
f(x) & =\sum_{a \in A_{p}} w_{a} x_{a}\left(1-\sum_{b \in A_{p} \backslash\{a\}} \overline{R I}_{p}(b, a) x_{b}\right) \\
& \geq \frac{1}{2} \sum_{a \in A_{p}} w_{a} x_{a} \geq \frac{1}{4 \beta_{p}} \text { opt. }
\end{aligned}
$$


Now, we show that $f(x)$ is increasing (not necessarily strictly) with the iterations during Phase 2. Consider a particular iteration and let $a$ be the link picked and then removed from $B$. If

$w_{a}\left(1-\sum_{b \in A_{p} \backslash\{a\}} \overline{R I}_{p}(b, a) x_{b}\right)>\sum_{b \in A_{p} \backslash\{a\}} \overline{R I}_{p}(a, b) w_{b} x_{b}$, then, $\frac{\partial f(x)}{\partial x_{a}}>0$ and hence increasing $x_{a}$ to 1 would increase $f(x)$; otherwise, $\frac{\partial f(x)}{\partial x_{a}} \leq 0$ and hence decreasing $x_{a}$ to 0 would also increase $f(x)$. So, in either case, $f(x)$ increases after each iteration during Phase 2.

Finally, we show that $I$ output at the end of Phase $\mathbf{3}$ is independent and its weight is at least $\frac{1}{4 \beta_{p}}$ opt. Let $z$ be the indicator vector of $I$, i.e., $z_{a}=1$ for each $a \in I$ and $z_{a}=0$ for each $a \in A_{p} \backslash I$. Then, for the vector $x$ in Phase 3, we have

$$
x_{a}\left(1-\sum_{b \in A_{p} \backslash\{a\}} \overline{R I}_{p}(b, a) x_{b}\right) \leq z_{a} \leq x_{a} .
$$

Consider any $a \in I$. Since

$$
\sum_{b \in A_{p} \backslash\{a\}} \overline{R I}_{p}(b, a) x_{b}<1,
$$

we have

$$
\begin{aligned}
& \sum_{b \in I \backslash\{a\}} \overline{R I}_{p}(b, a)=\sum_{b \in A_{p} \backslash\{a\}} \overline{R I}_{p}(b, a) z_{b} \\
\leq & \sum_{b \in A_{p} \backslash\{a\}} \overline{R I}_{p}(b, a) x_{b}<1 .
\end{aligned}
$$

Thus, $I$ is independent. In addition,

$$
\begin{aligned}
& \sum_{a \in I} w_{a}=\sum_{a \in A_{p}} w_{a} z_{a} \\
& \geq \sum_{a \in A_{p}} w_{a} x_{a}\left(1-\sum_{b \in A_{p} \backslash\{a\}} \overline{R I}_{p}(b, a) x_{b}\right) \\
& =f(x) \geq \frac{1}{4 \beta_{p}} \text { opt. }
\end{aligned}
$$

This completes the proof of the theorem.

Theorem 4.2 implies that LPWIS polynomial $4 \beta_{p^{-}}$ approximation algorithms for MWISL. By using the approximation-preserving reductions from MMF, MCMF and SFLS to MWISL developed in [13] we obtain polynomial $4 \beta_{p}$-approximation algorithms for MMF, MCMF and SFLS. Thus, Theorem 4.1 holds.

\section{Discussions}

In this paper, we have developed polynomial constantapproximation algorithms for four optimization problems MWISL, MMF, MCMF and SFLS in wireless networks under physical interference model with linear power assignment. It remains open whether these optimization problems have constant-approximation algorithms with other monotone and sublinear power assignments such as uniform power assignment and mean power assignment. A major technical obstacle to this open problem is that other power assignment of interest fail to have the constant-bounded inward local independence number in general. Indeed, both the design and analysis of the algorithms presented in Section IV of this paper is valid for any power assignment. Constant-approximation bound is possible for linear power assignment just due to the fact that the inward local independence number with linear power assignment is bounded by a constant. Thus, other networking parameters, as a counterpart to the inward local independence number, are expected to play essential roles for other power assignments. Despite of these challenges, the technical approach adopted in this paper is still promising. By slightly modifying the linear program in Phase 1 of the algorithm LPWIS, we can actually achieve a logarithmic approximation bounds for general monotone and sublinear power assignment, which have the same order as those achieved in [13] but have much smaller values.

ACKNOWLEDGEMENTS: This work was supported in part by the National Science Foundation of USA under grants CNS-0831831, CNS-0916666, and CNS-1219109, by the National Natural Science Foundation of P. R. China under grants 61128005 .

\section{REFERENCES}

[1] M. Andrews and M. Dinitz, Maximizing Capacity in Arbitrary Wireless Networks in the SINR Model: Complexity and Game Theory, IEEE INFOCOM 2009.

[2] D. Chafekar, V. Kumar, M. Marathe, S. Parthasarathy, and A. Srinivasan, Approximation algorithms for computing capacity of wireless networks with SINR constraints, IEEE INFOCOM 2008, pages 1166-1174.

[3] A. Fanghänel, T. Kesselheim, H. Räcke, and B. Vöcking, Oblivious interference scheduling, ACM PODC 2009.

[4] A. Fanghänel, T. Kesselheim, and B. Vöcking, Improved algorithms for latency minimization in wireless networks. In ICALP, July 2009.

[5] O. Goussevskaia, Y.A. Oswald, and R. Wattenhofer, Complexity in geometric SINR, Proc. of the 8th ACM MOBIHOC, pp. 100-109, September 2007.

[6] M. M. Halldórsson, Wireless Scheduling with Power Control, in ESA 2009, LNCS 5757, pp. 361-372, 2009.

[7] M. M. Halldórsson and P. Mitra, Wireless capacity with oblivious power in general metrics, SIAM SODA 2011: 1538-1548.

[8] M. M. Halldórsson and P. Mitra, Wireless capacity and admission control in cognitive radio, IEEE INFOCOM 2012: 855-863.

[9] M. M. Halldórsson and R. Wattenhofer, Wireless Communication is in APX. In ICALP, July 2009.

[10] T. Kesselheim, A Constant-Factor Approximation for Wireless Capacity Maximization with Power Control in the SINR Model, SIAM SODA 2011: 1549-1559.

[11] P.-J. Wan, Multiflows in Multihop Wireless Networks, ACM MOBIHOC 2009, pp. 85-94.

[12] P.-J. Wan, D. Chen, G. Dai, Z. Wang, and F. Yao, Maximizing Capacity with Power Control under Physical Interference Model in Duplex Mode, IEEE INFOCOM 2012.

[13] P.-J. Wan, O. Frieder, X. Jia, F. Yao, X.-H. Xu, S.-J. Tang, Wireless Link Scheduling under Physical Interference Model, IEEE INFOCOM 2011.

[14] P.-J. Wan, X. Jia, and F. Yao, Maximum Independent Set of Links under Physical Interference Model, WASA 2009.

[15] P.-J. Wan, C. Ma, S. Tang, and B. Xu, Maximizing Capacity with Power Control under Physical Interference Model in Simplex Mode, WASA 2011: 84-95.

[16] P.-J. Wan, X.-H. Xu, and O. Frieder, Shortest Link Scheduling with Power Control under Physical Interference Model, Proceedings of The 6th International Conference on. Mobile Ad-hoc and Sensor Networks (MSN'10), 2010. 